\title{
STRUCTURE PERFECTION DIAGNOSTICS OF SINGLE CRYSTALS BY MEANS OF DIFFRACTOMETRY MEASUREMENTS USING X-RAY CONTINUOUS SPECTRUM
}

\author{
L.I. Datsenko aND V.I. KiIRUPA \\ Institute of Semiconductor Plysics, National Academy of Sciences \\ Prosp. Nauki 45, 252028 Kiev, Ukraine
}

\begin{abstract}
Determination of the integral characteristics of structural perfection of a real crystal (i.e. Debye-Waller's static factor $\mathrm{e}^{-L}$ and coefficient of absorption $\mu_{\mathrm{ds}}$ due to diffuse scattering) is especially expedient using the suitably selected wavelengths of the $X$-ray continuous spectrum by investigation of the thickness $I(t)$, coordinate $I(x)$ as well as amplitude $I(W)$ dependencies of intensities at Laue or Bragg diffraction. Here $W$ is an amplitude of weak ultrasound vibrations excited in a sample for suppression of the Bragg component of reflectivity.
\end{abstract}

PACS numbers: 81.10.-z, 61.66.Bi, 61.72.Dd

\section{Introduction}

Structural defects are well known [1] to cause three main diffraction phenomena:

1. Displacement of the diffraction maximum relative to its position in an ideal crystal due to formation of an "averaged" lattice.

2. Its intensity attenuation due to deviation of atomic positions from this averaged lattice and to additional weakening of that value by diffuse scattering on defects.

3. Appearance of diffraction maximum diffuse tails.

The first effect is usually used by measurements of lattice parameters of a crystal which permits us to determine a mean value of an atomic volume change at the expense of defects. The second and third phenomena are described by means of the Debye-Waller factor $\mathrm{e}^{-L}$ as well as by coefficient of additional energy losses $\mu_{\mathrm{d} s}$ due to diffuse scattering $[1,2]$. These integral cliaracteristics of scattering permit us to describe quantitatively the structure perfection of a single crystal and give sometimes possibilities to determine the mean dimension $\bar{r}$ as well as concentration $\bar{n}$ of microdefects [3]. 
The unique properties of synchrotron radiation (i.e. wide spectrum wavelengths, polarization of electromagnetic waves in the accelerator orbit plane, high intensity of short-wave components) permit us to use it cfficicntly for single crystal structure diagnostics.

The aim of the present work consists of the suggestion of new approaches in structure diagnostic methods which have been developed by us in the last years, especially those realized under conditions of weak ultrasound excitation of a crystal. All these methods assume utilization of the continuous spectrum of wavelengths which facilitate determination of the mentioned parameters of structural perfection.

\section{Laue diffraction of $\mathrm{X}$-ray white radiation with wavelengths situated near the absorption $K$-edge}

The above-mentioned parameters of structural perfection, plus the concentration of semiconductor compound components [4] as well as deviation of such substances from stoichiometric composition [5] can be determined from values of the intensity jumps (ratios) $S=i_{2} / i_{1}$ measured for two wavelengths near the $K$-edge of absorption $\left(\lambda_{K}\right)$ of a substance. $i_{2}$ and $i_{1}$ are the intensities measured in Laue diffraction in the long-wave $\left(\lambda_{2}>\lambda_{K}\right)$ and short-wave $\left(\lambda_{1}<\lambda_{K}\right)$ regions near $\lambda_{K}$. Let us note that there is a considerable eflect of dislocation density $N_{\mathrm{d}}$ in Ge on the value of $S$ as the example of efficiency of such approach (Fig. 1). One can see that values of the $i_{2}$ and $i_{1}$ intensities are noticeably changed near

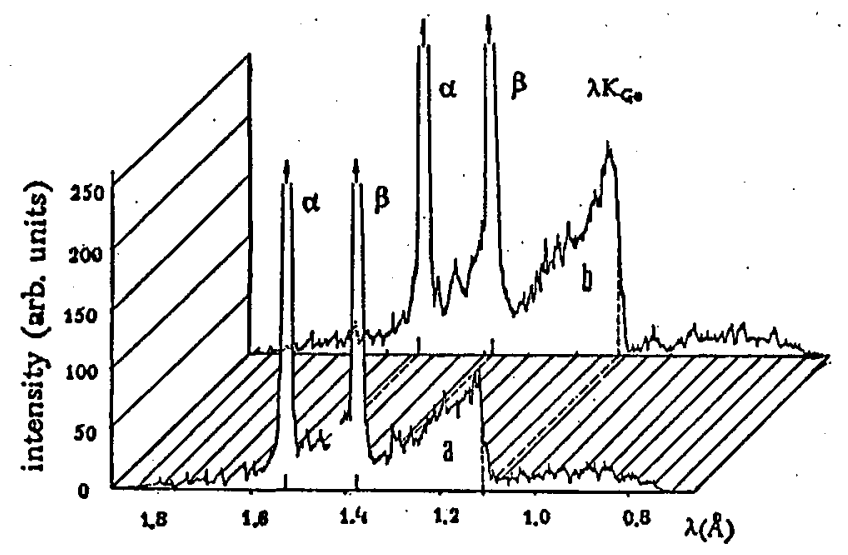

Fig. 1. Lauc-diffracted X-ray continuous spectrum near the absorption $K$-edges of the Ge specimen with dislocation. (a) Dislocation density $N_{\mathrm{d}}=0$, (b) $N_{\mathrm{d}}=2.4 \times 10^{5} \mathrm{~cm}^{-2}$.

$\lambda_{K \mathrm{Ge}}=1.116 \AA$. Considering the graph of the thickness dependence of the intensities ratio logarithm $\ln (S)=f(t)$ one can determine $\exp (-L)$ for a crystal with dislocations [3]:

$$
\exp (-L)=\left(1-A_{\mathrm{d}} Z\right) /\left(1-A_{\mathrm{p}} Z\right)
$$


where $A_{\mathrm{d}}$ and $A_{\mathrm{p}}$ are the slopes of those dependencies for a crystal with dislocations and without them respectively. $Z=\gamma_{1} \gamma_{2}\left(\mu_{1} \gamma_{2}-\mu_{2} \gamma_{1}\right), \mu_{1}$ and $\mu_{2}$ are normal photoelectric coefficients of absorption for the wavelengths $\lambda_{1}$ and $\lambda_{2}$ and $\gamma_{1,2}=\cos \theta_{1,2}$ for those wavelengths. The value $L$ for a Ge sample with $N_{\mathrm{d}} \approx 2.4 \times 10^{5} \mathrm{~cm}^{-2}$ happened to be $\approx 4.1 \times 10^{-2}$ which is in agreement with results of independent measurements. One should note that $L$ can be also calculated using data of the metallographical dislocation density $N_{\mathrm{d}}^{\mathrm{m}}$ according to [6]:

$$
L=(\boldsymbol{H} \cdot b) \Lambda^{2} N_{\mathrm{d}}^{\mathrm{m}} / 4 \pi .
$$

Ilere $A, \boldsymbol{H}$ and $\boldsymbol{b}$ stand for the extinction length, the diffraction vector and the Burgers vector of dislocation respectively.

Measurements of integral reflectivities near the $\mathrm{Ga}, \mathrm{As}$ and $\mathrm{Ge} K$-edges of absorption permitted us to determine the type of defects formed during the solid solution decay of $\mathrm{Te}$ in GaAs crystals and $\mathrm{Li}$ in Ge samples as well [3].

\section{Laue diffraction of the continuous spectrum wavelengths under condition of $\mathrm{X}$-ray acoustic interactions}

The amplitude dependence of the reflectivities $I_{\mathrm{d}}(W)$ and $I_{\mathrm{p}}(W)$ measured in a weakly absorbing $\mathrm{Si}$ crystal with defects (d) and without them (p) under conditions of acoustic excitation were used in [6] for determination of the $L$ and $\mu_{\mathrm{ds}}$ parameters

$$
\begin{aligned}
& L=\frac{\left\langle I_{\mathrm{d}}^{*}-I_{\mathrm{p}}^{*}\right\rangle \mathrm{e}^{-z}}{I_{\mathrm{p}}} \frac{A}{4 \pi^{2}} \frac{Z}{\left(1-\mathrm{e}^{-z}\right)} \sum_{\sigma, \pi} \mathrm{e}^{\varepsilon \varepsilon h}, \\
& \mu_{\mathrm{ds}}=-\frac{\gamma}{t} \ln \frac{I_{\mathrm{d}}-I_{\mathrm{d}}^{*}}{I_{\mathrm{p}}-I_{\mathrm{p}}^{*}}
\end{aligned}
$$

where $W$ is amplitude of ultrasound vibrations $(W \approx 0.1 \AA)$. $I_{\mathrm{d}, \mathrm{p}}^{*}$ characterize the minimum values of the $I(W), z=\mu_{\mathrm{ds}} t / \gamma, h=\mu t / \gamma$ and $\varepsilon$ is the ratio of imaginary parts of the Fourier coefficients of susceptibility $\chi\left(\varepsilon=\chi_{i h} / \chi_{i 0}\right)$. Summation of intensities in Eq. (3) is performed for $\sigma$ - and $\pi$-states of polarization $(c=1$ or $\left.c=\cos \left(20_{B}\right)\right)$. The principal idea of such experiments consists in suppression of the coherent component of reflectivity by ultrasound which gives the unique possibility to analyze separately the diffuse component and its dependence on the integral characteristics of a given reflection.

Now we have suggested to choose a specific wavelength $\lambda_{0}$ of the continuous spectrum for which the intensities of a real and perfect crystal coincide $\left(I_{\mathrm{d}}=I_{\mathrm{p}}\right)$ due to equality of scattering and absorption contribution to total reflectivity of a sample with structural defects [3]. Under this condition one can use only one dependence $I_{\mathrm{d}}(W)$ measured in a real crystal as distinguished from [6] where such a graph was plotted for a sample with perfect structure, too.

The measurement procedure and the formulas (3) are considerably simplified by such an approach without any loss of information quality

$$
L=\frac{I_{\mathrm{N}}-2 \exp (c \varepsilon h) \exp (-z)}{1-2 \exp (-c \varepsilon h)} \frac{\Lambda}{4 \pi^{2} \iota} \frac{Z}{1-\exp (-z)} \sum_{\sigma, \pi} \exp (c \varepsilon h)
$$




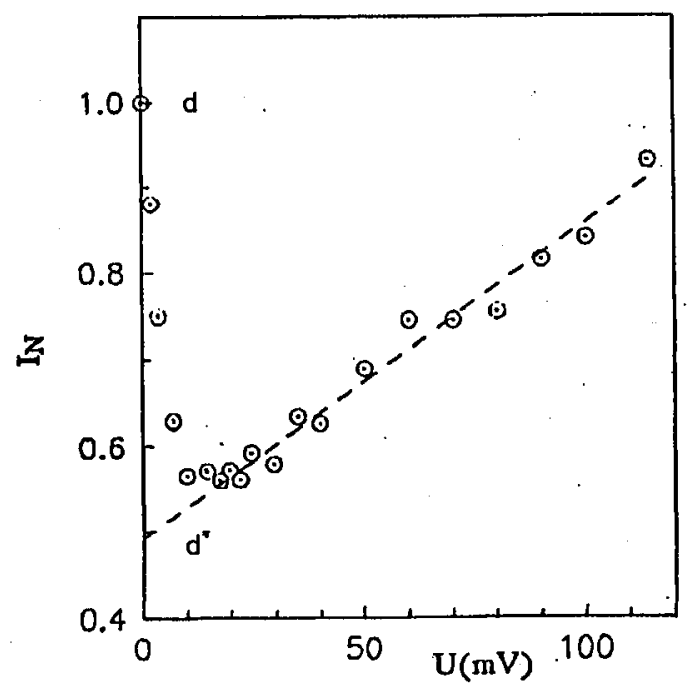

Fig. 2. The normalized intensity $I_{N}(W)$ as a function of the voltage $U$ on a transducer for an ultrasound excitable $\mathrm{Si}$ crystal with structural defects measured at the 220 Laue reflection by means of continuous spectrum radiation $(\lambda=0.4 \AA)$.

$$
\mu_{\mathrm{ds}}=-\frac{\gamma}{t} \ln \frac{1-I_{\mathrm{N}}}{1-2 \exp (-c \varepsilon h)},
$$

where $I_{\mathrm{N}}=I_{\mathrm{d}}^{*} / I_{\mathrm{d}}$ is the intensity normalized to the initial state $(W=0)$.

We determined according to (4) the integral claracteristics $L=1.7 \times 10^{-3}$ and $\mu_{\mathrm{ds}}=0.5 \mathrm{~cm}^{-1}$ for the Si crystal with thickness $t=1 \mathrm{~cm}$ containing microdefects after measurements of $I_{\mathrm{N}}$ as a function of the voltage $U$ on a transducer ( $W$ is proportional to $U$ ) (Fig. 2) at a wavelength $\lambda_{0}=0.404 \AA$. It permitted us to calculate according to [2] the average dimension $\tilde{r}$ and concentration $\bar{n}$ of microdefects $\left(\bar{r}=1.5 \mu \mathrm{m} ; \bar{n}=5 \times 10^{3} \mathrm{~cm}^{-3}\right)$ formed during high temperature decay of the oxygen solid solution. The dislocation density was also determined by such methods in another Si crystal. The procedure of intensity measurements and conditions of ultrasound excitation were similar to those in the previous paper [6].

\section{Analysis of a spatial distribution of diffiacted beam intensity in the Bragg case}

The method of spatial (not angular) distribution of a diffracted beam intensity was developed in [7-9] for reflection from the surface of a crystal. The necessary condition for observation of diffuse scattering by defects situated within the absorption depth $\mu^{-1}$ below the surface of a crystal is the requirement $\mu^{-1}>\Lambda$ which is strictly fulfilled for hard radiation. When the divergence of the incident beam exceeds (somewhat) the half-width of a diffraction maximum, this radiation penetrates to the absorption depth in the sample and one can observe the extended diffuse tail as a function of coordinate $x$ along the surface of a crystal. 
Investigation of the coordinate distribution $I(x)$ of such intensity permits us to determine the $L$ parameter [7-9]:

$$
L=0.5\left[I(x) / I_{0}\right] \exp \left(\mu t / \cos O_{B}\right),
$$

where $I_{0}$ is the peak intensity of the difraction maximum. Since there are always hard components of radiation in the continuous spectrum, the necessary conditions for observation of diffuse scattering are strictly fulfilled. The coordinate dependence of the $L(x)$ parameter beginning from the place of the 555 diffraction maximum calculated with the formula (5) for a wavelength $\lambda=0.4 \AA$ is shown in Fig. 3. The lower curve relates to the initial state of the sample $\left(L=0.5 \times 10^{2}\right)$.

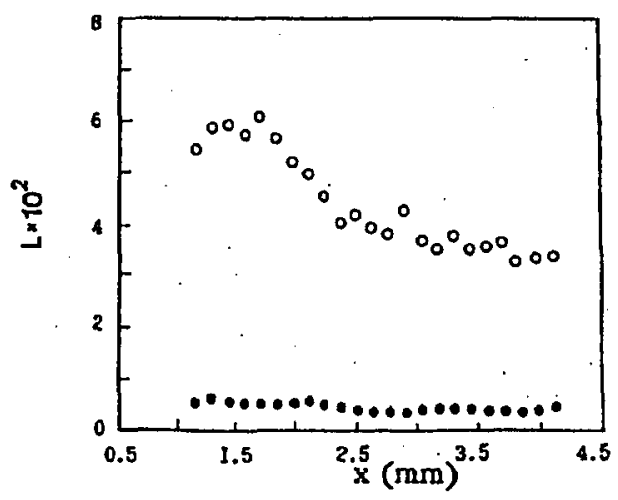

Fig. 3. Coordinate dependencies of the Debye-Waller factor $L(x)$ obtained in a Si crystal with structure defects by analysis of spatial distribution of the Bragg diffracted beams. - initial state, $\circ$ after treatment at $1100^{\circ} \mathrm{C}(6 \mathrm{~h})$.

The upper one relates to its state after heat treatment for $6 \mathrm{~h}$ at $1100^{\circ} \mathrm{C}$. One can see that inhomogeneous defect structure formed during such thermal treatment $\left(L \approx 5 \times 10^{-2}\right.$ ). Such structure is probably comnected with peculiarities of the oxygen distribution in $\mathrm{Si}$.

\section{Conclusion}

Using of the X-ray continuous spectrum permits us to choose the wavelengths which are desirable not only for precision measurements of the lattice parameter $\left(\theta_{\mathrm{B}} \rightarrow \pi / 2\right)$ but to provide the necessary conditions for application of the new nondestructive methods of structure diagnostics of crystals. The values of the integral characteristics of structure perfection $\left(L, \mu_{\mathrm{ds}}\right)$ can be determined from the thickness, coordinate and amplitude dependencies of intensity. The data obtained are in agreement with results of independent difractometry measurements. 


\section{Acknowledgments}

This work was partially supported by the Ukrainian Committee for Scientific Research and Technology.

\section{References}

[1] M.A. Krivoglaz, The Theory of X-ray and Neutron Scallering by Real Crystals, Nauka, Moskva 1967, p. 336.

[2] P.H. Dederichs, Phys. Rev. B 1, 1306 (1970).

[3] L.I. Datsenko, V.B. Molodkin, M.E. Osinovskii, Dynamical Scaltering of X-Rays by Real Crystals, Naukova Dumka, Kíev 1988, p. 198.

[4] V.P. Klad'ko, A.N. Gureev, L.I. Datsenko, K.P. Kurbanov, A.V. Fomin, Kristallografiya 32, 1202 (1987).

[5] L.I. Datsenko, V.P. Klad'ko, T.G. Kryshtab, in: Proc. 81 Int. School on Defects in Crystals, Szczyrk (Poland) 1988, World Scientific, Singapore 1988, p. 59.

[6] I.R. Entin, V.I. K’hrupa, L.I. Datsenko, J. Appl. Crystallogr. 23, 355 (1990).

[7] I.R. Entin, V.I. Khrupa, J. Appl. Crystallogr. 24, 403 (1991).

[8] V.I. Khrupa, I.R. Entin, Mclallofizika 13, 117 (1991).

[9] V.V. Nikolaev, V.I. Khrupa, Metallofizika 14, 72 (1992). 